\title{
Human islet transplantation network for the treatment of Type I diabetes: first data from the Swiss-French GRAGIL consortium (1999-2000)
}

\author{
P.Y. Benhamou ${ }^{1}$, J. Oberholzer ${ }^{2}$, C. Toso ${ }^{2}$, L. Kessler ${ }^{3}$, A.Penfornis ${ }^{4}$, F. Bayle ${ }^{1}$, C. Thivolet ${ }^{6}$, X. Martin ${ }^{5}$, F.Ris ${ }^{2}$, \\ L. Badet ${ }^{5}$, C. Colin ${ }^{7}$, P. Morel ${ }^{2}$ on behalf of the GRAGIL group*
}

${ }^{1}$ Department of Endocrinology, University Hospital, Grenoble, France

${ }^{2}$ Department of Surgery, University Hospital, Geneva, Switzerland

${ }^{3}$ Department of Endocrinology, University Hospital, Strasbourg, France

${ }^{4}$ Department of Endocrinology, University Hospital, Besançon, France

${ }^{5}$ Department of Urology, Hospices Civils, Lyon, France

${ }^{6}$ INSERM U449, Lyon, France

${ }^{7}$ Department of Medical Information, Hospices Civils, Lyon, France

\section{Abstract}

Aims/hypothesis. Improvements in islet transplantation require clinical series large enough to implement controlled new strategies. The goal of this study was to demonstrate the feasibility of a multicentre network for islet transplantation in Type I (insulin-dependent) diabetic patients.

Methods. The five centres (Besançon, Geneva, Grenoble, Lyon, Strasbourg) of the GRAGIL network allow pancreas procurement, recipient recruitment, transplantation procedure and follow-up. Islet isolation is, however, performed in one single laboratory (Geneva). Pancreata were procured in each of the five centres and transported to Geneva with an ischaemia time of less than 8 hours. Islets were isolated using a standard automated method. If the islet number was too low for a graft ( $<6000$ Islet-equivalent / $\mathrm{kg}$ ), islets were cultured up to 12 days until another isolation was possible. Islets were transplanted by percutaneous transhepatic intraportal injection. Immunosuppression consisted of cyclosporine, myco- phenolate mofetil, steroids and an anti-interleukin 2 receptor antibody.

Results. From March 1999 to June 2000, 56 pancreata procurements were performed with an average yield of 234500 islet-equivalent, with 32 preparations over 200000 islet-equivalent. Ten C-peptide negative Type I diabetic patients ( 5 men and 5 women, median age 44 years, median diabetes duration 29 years) with an established kidney graft ( $>6$ months) received $9030 \pm 1090$ islet-equivalent $/ \mathrm{kg}$ with a median purity of $63 \%$. The number of pancreata required for each graft was $1(\mathrm{n}=5)$ or $2(\mathrm{n}=5)$. At the completion of a 12 month follow-up, we observed $0 \%$ primary nonfunction, $50 \%$ graft survival and $20 \%$ insulin-independence.

Conclusions/interpretation. This study demonstrates the interest and the feasibility of a multicentre collaboration in human islet transplantation. [Diabetologia (2001) 44: 859-864]

Keywords Pancreatic islet transplantation, Type I diabetes mellitus, multicentre network.
Received: 30 November 2000 and in revised form: 23 March 2001

Corresponding author: Dr Pierre Y. Benhamou, Department of Endocrinology, CHU, BP 217X, 38043 Grenoble, France, E-mail: benhamou@ujf-grenoble.fr

*The GRAEIL Group is listed in the Acknowledgements

Abbreviations: IEQ, Islet-equivalent; CMV, cytomegalovirus; JAK, islet after kidney; HLA, human leucocyte antigen; GAD, glutamate acid decarboxylase.
Islet transplantation could be used to treat Type I (insulin-dependent) diabetes mellitus. Until now, this experimental procedure has been restricted to patients with end-stage renal failure, with a kidney graft or awaiting one. In this group of patients with an advanced stage of diabetic disease, the results of islet transplantation have been modest. By the end of 1999, the insulin-independence rate reported in the International Registry was $8 \%$ a year [1]. However the most active transplantation centres reached an average of $20 \%$ insulin-independence and 50 to $70 \%$ islet function rate [1-5].

Recently reported data has given fresh impetus to islet transplantation [6]. The achievement of $100 \%$ 
insulin-independence in a consecutive series of seven non-uraemic patients represents a major breakthrough. None of these patients had kidney failure but were included because of severe metabolic disturbances (the so-called brittle diabetes). This study also highlights the critical role of islet mass in the success of the procedure because all the patients required at least two donor pancreata and an average of 11000 islet equivalents for each kilogram of body weight.

One question raised by this pioneering study, is how to supply all potential diabetic candidates with allogeneic islets. The current pancreas transplantation waiting lists underscore the donor crisis that islet transplantation teams will face, especially for brittle diabetic patients who need two donor organs. There are, however, a large number of non-procured organs in many countries, but before in vitro expansion of islet tissue is made clinically available, organizational improvements are needed to address organ shortages for allotransplantation.

Our study aimed to demonstrate the feasibility of a multicentre network model such as GRAGIL, for islet transplantation in Type I diabetic patients. Comprising five Transplant Centres (Besançon, Geneva, Grenoble, Lyon, Strasbourg), this network permitted pancreas procurement, recipient recruitment, transplantation procedure and follow-up in each individual centre although islet isolation was done in one single laboratory (Geneva). Our study indicates that this regional organization is an efficient way to implement islet transplantation and make it available for large groups of diabetic patients.

\section{Methods}

The GRAGIL network. The University Hospitals of Besançon, Geneva, Grenoble, Lyon and Strasbourg agreed to participate in the study, formalized in a convention and approved by the French Transplantation Agency (Etablissement Français des Greffes) and National Health Department (Direction Générale de la Santé), and named GRAGIL (Groupe de Recherche Rhin Rhône Alpes Genève pour la transplantation d'Ilots de Langerhans or in English The Rhine Rhone Alpes Geneva Research Group for Islet Transpantation).

Patients. Adult Type I diabetic patients were included in this study if they fullfilled the criteria of a negative $(<0.2 \mathrm{ng} /$ $\mathrm{ml}$ ) basal and glucagon-stimulated serum C-peptide and an established kidney transplantation functioning for over 6 months (glomerular filtration rate $>40 \mathrm{ml} / \mathrm{min}$ ). The protocol was approved by the ethics board of Grenoble and Geneva University Hospitals and each patient gave their written informed consent. Following inclusion, all patient's clinical and immunological information was mailed to the Geneva centre and posted on a secure Intranet Web for the rest of the investigators to see. Until transplantation, a patient's file was updated every 3 months with clinical observations and centralised glycated haemoglobin. Serum was sent out to every investigation centre for future pretransplant crossmatch determinations.
Pancreas procurements. Pancreata were procured from cadaveric, heart-beating donors and offered to every centre of the GRAGIL network according to a standardized procedure. When a multiorgan procurement was set, the local transplant coordinator would call the Geneva islet isolation laboratory and fax a donor information checklist. Donors were selected according to the following criteria.

Major criteria for donors were: 20-60 years of age; preserved haemodynamics (no sign of splanchnic underperfusion, such as high amine needs, functional renal failure or high liver transaminases) ; blood glucose less than $11.1 \mathrm{mmol} / \mathrm{l}$; no history of chronic pancreatitis.

Minor criteria were: BMI of $25 \mathrm{~kg} / \mathrm{m}^{2}$ or more or body weight of $50 \mathrm{~kg}$ or more; hospitalisation of less than 7 days; normal serum amylase and lipase ; no history of alcohol abuse.

Pancreata were removed en bloc following cold perfusion with University of Wisconsin solution and shipped by ambulance to Geneva. A cold ischaemia time of less than $8 \mathrm{~h}$ was mandatory in the protocol and feasible, as Geneva is within 3 hours driving distance of every one of the four French centres of the network.

Islet preparation. On arrival at Geneva islet isolation facilities, the pancreas was distended by the intraductal injection of an enzymatic solution (Liberase, Roche Diagnostics, Meylan, France) and digested at $37^{\circ} \mathrm{C}$ for 20 to $30 \mathrm{~min}$ as previously described [5]. Islets were purified by isopycnic centrifugation in a discontinuous Euro-Ficoll gradient with a refrigerated cell separator (COBE 2991, Cobe Laboratories, Lakewood, Colo., USA). The number of islets was measured using a sample after dithizone staining and expressed as the equivalent number of islets (IEQ: number of islets if all were $150 \mu \mathrm{m}$ in diameter) [7]. Islet counting was carried out on the day of isolation and repeated on the day of transplantation. Islets were cultured at $24^{\circ} \mathrm{C}$ in CMRL 1066 (Gibco BRL, Cergy Pontoise, France) containing $10 \%$ fetal calf serum. When an appropriate preparation was available, it was processed for transplantation as soon as possible, i. e. the next day when the islet count was sufficient or within 14 days when a second islet preparation was needed to reach the $6000 \mathrm{IEQ} / \mathrm{kg}$ threshold stated in our protocol. In the latter case, islets were first cultured during a maximum of 7 days at $24^{\circ} \mathrm{C}$, then for another maximum of 7 days at $37^{\circ} \mathrm{C}$, as previous studies had indicated that the immunomodulatory effects of low temperature culture beyond this period were challenged with a deleterious impact on islet viability and function [8]. Islet functionality was determined by static incubation within $24 \mathrm{~h}$ before transplantation, and viability was checked by microscopic morphological assessment. Transplanted islets were washed and collected in 50-ml syringes in $\mathrm{X}$-Vivo medium (Bio Whittaker, Emerainville, France) containing $4 \%$ human albumin and shipped by ambulance at ambient temperature to the transplantation centre. Preliminary experiments done in our laboratory did not show any deleterious effects of short-term $(4 \mathrm{~h})$ maintenance of islets in this medium at room temperature in static incubation assays, and in regard to viability and degree of apoptosis.

Islet transplantation. Transplantation criteria were set as follows: an islet mass threshold of $6000 \mathrm{IEQ} / \mathrm{kg}$; purity of more than $50 \%$; functionality reaching a stimulation index of more than 2. Each islet transplantation should take into account the recipient's $\mathrm{ABO}$ blood type or respect $\mathrm{AB} 0$ compatibility. HLA repeats with the established kidney graft were avoided. HLA matching was not mandatory, and this criterion was only taken into account when there were several potential recipients. All transplantation proposals were issued by the Geneva immunologists according to the centralized recipient database. Do- 
nor tissue was crossmatched with potential recipient's serum for lymphocytotoxic antibodies in the procurement centre.

Islet collection, transport and recipient conditioning were synchronized in order to minimize the risk of islet injury. All islet preparations were pooled and transplanted in a one-step procedure. Recipients were sedated with $100 \mathrm{mg}$ hydroxyzine (UCB Pharma, Nanterre, France) and $0.5 \mathrm{mg}$ alprazolam (Pharmacia Upjohn, Guyancourt, France) and received a local anaesthesia with ropivacaine (Astra, Rueil-Malmaison, France). Islet transplantation was done by a percutaneous transhepatic approach which allowed the portal vein to be catheterized under ultrasound guidance. Islets were slowly infused over an average period of $30 \mathrm{~min}$ with portal venous pressure monitored throughout the procedure. A $5000 \mathrm{U}$ bolus of heparin was administrated through the portal catheter before transplantation and gelatin-sponge particles (Gelfoam) were embolized into the intraparenchymal track during catheter removal.

Immunosuppression, adjuvant therapy and follow-up. Recipients were conditioned by basiliximab (Simulect, Novartis) given at a dose of $20 \mathrm{mg}$ IV on day 0 and +4 , and methylprednisolone given at a dose of $1 \mathrm{~g}$ IV on day 0 and $100 \mathrm{mg}$ IV on day +1 . Oral prednisone was tapered down from a dose of $20 \mathrm{mg} / \mathrm{d}$ from day +2 until day +14 , to a dose of $15 \mathrm{mg} / \mathrm{d}$ until the end of the first month and a dose of $10 \mathrm{mg} / \mathrm{d}$ until the end of the sixth month. Cyclosporin (Neoral, Novartis) was given twice a day at a dose of $5 \mathrm{mg} / \mathrm{kg} / \mathrm{d}$ aiming at a trough level of $150-200 \mathrm{ng} / \mathrm{ml}$ during the first month and $100-150 \mathrm{ng} / \mathrm{ml}$ thereafter. Mycophenolate mofetil (Cellcept, Roche) was given orally at a dose of $2 \mathrm{~g} / \mathrm{d}$. Two patients on tacrolimus (Prograf, Fujisawa) following kidney transplantation were maintained under this regimen at a dose of $2-3 \mathrm{mg}$ daily aiming at a trough level of $6(4-8) \mathrm{ng} / \mathrm{ml}$.

Similarly to the Giessen Protocol described previously, adjuvant therapy consisted of nicotinamide $(2 \mathrm{~g} / \mathrm{d})$, vitamin $\mathrm{E}$ $(400 \mathrm{mg} / \mathrm{d})$, verapamil $(240 \mathrm{mg} / \mathrm{d})$ given until the end of the sixth month and pentoxyfylline $(1600 \mathrm{mg} / \mathrm{d})$ given until the end of the $1^{\text {st }}$ month [9]. Rocephin ( $2 \mathrm{~g}$ IV) was given prophylactically on the day of transplantation. CMV prophylaxis was performed with ganciclovir intravenously (Roche), $5 \mathrm{mg} / \mathrm{kg}$ bodyweight bid for 14 days, or valaciclovir orally, $800 \mathrm{mg} / \mathrm{day}$, for 3 months, in case of negative CMV serology in the recipient and positive CMV-serology in the donor. CMV-early antigen in peripheral blood leukocyte was monitored during the first 6 months after transplantation.

Insulin therapy was maintained unless the patient experienced hypoglycaemia. Recipients were switched to continuous IV regular insulin during the first week, targeting a blood glucose concentration between 4 and $8 \mathrm{mmol} / \mathrm{l}$, then intensive insulin therapy was given as required.

Evaluation was made on a monthly basis by determining fasting or glucagon-stimulated serum C-peptide (C-PEP-CT2 kit, CIS bio international, Gif sur Yvette, France), mean blood glucose concentrations and $\mathrm{HbA}_{1 \mathrm{c}}$ values as determined by high performance liquid chromatography (normal values: 4-6\%). Islet cell antibodies were measured by a standardized indirect immunofluorescent assay. The presence of GAD65 and IA-2 antibodies was detected by radioligand assays using plasmid cDNA encoding full length human GAD65 and the cytoplasmic part (aminoacids 603-979) of human IA2. All biological measurements were centralized within the network. Blood glucose was monitored by memory capillary glucose meters using InTouch software (Lifescan).

Study design. The study was carried out as a two-stage phase I-II trial with a sample size of 9 patients in the first stage (ex- pected efficacy defined by a C-peptide secreting graft set at $30 \%$ ) and 12 patients in the second stage according to the Gehan design [10].

\section{Results}

Procurement activity. Procurement activity within GRAGIL began in March 1999. Between March 1 1999 and June 1 2000, a total of 56 pancreata were procured and sent to Geneva. These organs originated from Swiss $(n=33)$ and French $(n=23)$ centres. During that period, 116 pancreata were offered for islet isolation but 60 were rejected because they did not meet the criteria mentioned above. The average yield was $234500 \pm 178764$ IEQ (range 0-1100000), with 32 preparations reaching an arbitrary threshold of 200000 IEQ. Average secondary warm ischaemia and cold ischaemia times were $50.6 \pm 20$ min (range 20-85 $\mathrm{min}$ ) and $6.1 \pm 1 \mathrm{~h}$ (range $3.5-7.5 \mathrm{~h}$ ), respectively. Secondary warm ischaemia time was defined as the time from aortic clampage until pancreas retrieval. From these 56 preparations, 20 were used for a transplantation: 9 islet-after-kidney transplantations were performed, using 14 islet preparations (4 single-donor and 5 dual-donor transplantations). During the same period, 4 simultaneous-islet-kidney transplantations were performed in the Geneva centre, using 6 of the available 56 islet preparations, but these are not described here.

Characteristics of the patients and the grafts. Between March 11999 and June 1 2000, nine islet-after-kidney transplantations were performed. An additional IAK transplantation performed in Geneva in November 1998 was included in this series because it was performed according to the procedure and monitoring protocol of GRAGIL albeit before pancreas exchanges were legally authorized within GRAGIL. The 10 recipients were 5 men and 5 women (median age 44 years, range 28-62) with Type I diabetes for a median of 29 years (range 15-42) and with an established kidney allograft for 5 years (range 2-9) (Table 1). Two recipients (identification numbers (ID) \# 3 and \#7) were maintained on immunosuppression with tacrolimus.

The patients received transplantations with a mean $( \pm$ SD) number of islets of $9030 \pm 1090$ isletequivalents per $\mathrm{kg}$ of recipient's body weight (range $4500-17300$ ) and an average purity of $63 \%$ (range 50-90). Islet preparations were cultured for an average of 2 days (range 1-12) before transplantation (Table 2). In vitro, the stimulation index of the transplanted islet preparations in the static glucose incubation assay was means $6.3 \pm 3 \mathrm{SD}$. No patient exhibited any islet-cell, anti-GAD65 nor IA-2 antibody before transplantation, except patient \#9 who had a low titre of IA-2 antibody before transplantation (titre $4.4 \mathrm{U}$ ). 
Table 1. Characteristics of recipients

\begin{tabular}{|c|c|c|c|c|c|c|}
\hline Patient ID & $\begin{array}{l}\text { Follow-up }{ }^{a} \\
\text { (months) }\end{array}$ & City & Sex & Age (years) & $\begin{array}{l}\text { Diabetes } \\
\text { duration (years) }\end{array}$ & $\begin{array}{l}\text { Kidney graft } \\
\text { duration (years) }\end{array}$ \\
\hline 1 & 27 & Geneva & M & 62 & 36 & 8 \\
\hline 2 & 22 & Grenoble & M & 39 & 26 & 2 \\
\hline 4 & 18 & Strasbourg & $\mathrm{M}$ & 52 & 24 & 4 \\
\hline 5 & 17 & Geneva & M & 47 & 33 & 5 \\
\hline $6^{\mathrm{b}}$ & 15 & Geneva & $\mathrm{F}$ & 50 & 42 & 9 \\
\hline $9^{b}$ & 12 & Grenoble & M & 38 & 28 & 8 \\
\hline 10 & 12 & Lyon & $\mathrm{F}$ & 41 & 30 & 6 \\
\hline Median & 16 months & & $5 \mathrm{M} / 5 \mathrm{~F}$ & 44 years & 29 years & 5 years \\
\hline
\end{tabular}

${ }^{a}$ As of February 2001 - (Shaded areas show failed transplantations)

${ }^{\mathrm{b}}$ Patients achieving insulin-independence

Table 2. Characteristics of Islet Grafts

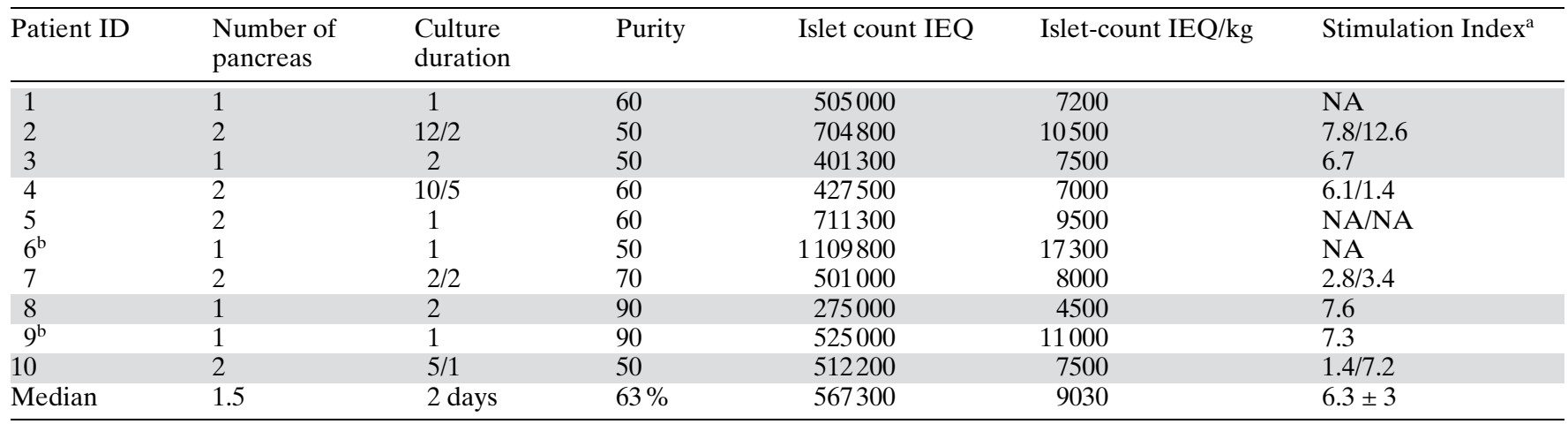

Shaded areas show failed transplantations

a Stimulation index was defined as the ratio of insulin secretion measured under stimulated conditions (glucose $20 \mathrm{mmol} / \mathrm{l})$ and basal conditions (glucose $3 \mathrm{mmol} / \mathrm{l}$ )

${ }^{\mathrm{b}}$ Patients achieving insulin-independence

The mean delay between the completion of islet collection in Geneva and the initiation of islet portal infusion was 4 hours for the French centres and 1 hour for Geneva. Tolerance of the procedure was good and no important change in portal pressure occurred. Liver function tests remained normal, although a slight and reversible increase in transaminases was observed in some patients. Three patients experienced a perihepatic haematoma which evolved favorably within a week.

Outcome of the grafts. All recipients showed an immediate function of their graft, as defined as the detection of more than $0.5 \mathrm{ng} / \mathrm{ml}$ serum C-peptide according to International Islet Registry criteria. Five patients gradually lost graft function $(\mathrm{C}$-peptide $<0.5$ $\mathrm{ng} / \mathrm{ml}$ ) after 2 to 10 months. The five other patients continued to have a functioning islet graft with a follow-up of 12 months. Two patients became insulin-independent between 6 and 8 months after transplantation and remained so with a follow-up of 12 months. In the three other C-peptide positive patients, insulin requirements decreased from $56 \pm 10 \mathrm{U} /$ day to $30 \pm 18 \mathrm{U} /$ day after 6 months. $\mathrm{HbA}_{1 \mathrm{c}}$ levels were reduced in these five patients from $8.6 \pm 1.6 \%$ to $6.0 \pm 0.4 \%$. Metabolic data are summarized in Table 3 .

As of February 2001, 8 of the 10 recipients had no increase in islet cell, GAD nor IA-2 antibodies following transplantation. The patient with identification number \#9 had positive IA-2 antibodies before transplantation (titre $4.4 \mathrm{U}$ ) that were measured after 1 month (3 U) and after 3 months ( $8 \mathrm{U}$ ). Patient \#8 developed anti-GAD antibodies (titre $0.42 \mathrm{U}$ ) and IA-2 antibodies (titre 1.6U) and lost graft function 3 months after transplantation. Patient \#10 also had positive islet cell (titre $16 \mathrm{U})$, IA-2 (12 U) and GAD65 (0.71 U) antibodies following transplantation and lost graft function after the first month.

Kidney function has remained stable in our series of patients up to the time of this paper. 
Table 3. Metabolic-outcome of recipients of islet grafts

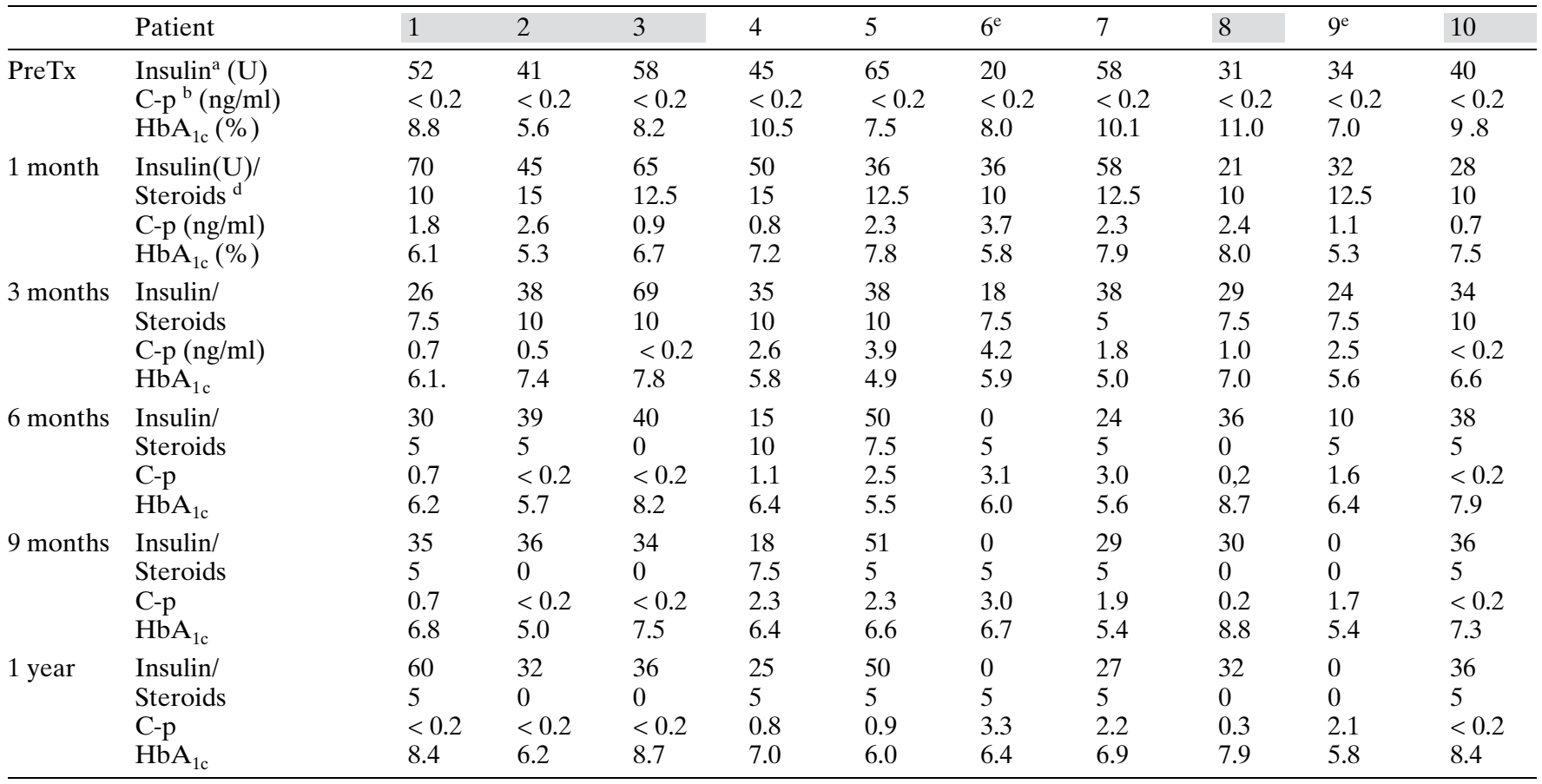

${ }^{\mathrm{a}}$ Units/day; ${ }^{\mathrm{b}} \mathrm{ng} / \mathrm{ml} ;{ }^{\mathrm{c}} \%$; ${ }^{\mathrm{d}} \mathrm{mg}$ prednisone/day

${ }^{\mathrm{e}}$ Patients achieving insulin-independence (Shaded areas show failed transplantations)

C-p = C-peptide

\section{Discussion}

By June 2000, the procurement and transplantation activities of the GRAGIL network had been in place for 15 months. During this time period, 56 pancreata were processed for islet isolation. Altogether 9 islet-afterkidney transplantations and 4 simultaneous-islet-kidney transplantations (data not shown) were performed. The overall metabolic results of IAK transplantations, assessed with a follow-up of 12 months for every patient, including $10^{\text {th }}$ transplantation previously performed under the same protocol, show a $0 \%$ primary nonfunction, a $50 \%$ graft survival rate (as defined by a basal serum C-peptide $\geq 0.5 \mathrm{ng} / \mathrm{ml}$ ) and a $20 \%$ insulinindependence rate. The International Registry data showed an $8 \%$ insulin-independence rate and a $31 \%$ graft survival rate [1]. Thus our network achieved the same results as those reported by single centres in the International Registry.

The average islet isolation yield in this series (234500 IEQ) is similar to those published by others [11]. From 56 pancreata processed, only 20 were used for islet transplantation. However, 32 preparations reached an arbitrary threshold of 200000 IEQ that could have been used in a sequential transplantation setting as previously proposed $[6,12]$. These islet isolation results were obtained by applying stringent donor selection criteria which reduced, however, the number of potentially available pancreata from 116 to $56[11,13,14]$.
On the other hand, the value of these donor selection criteria for the purpose of predictions is low. It is probable that several pancreata rejected on our criteria could have resulted in good islet yields. In addition, active pancreas transplantation programmes in three of five GRAGIL centres competed with this islet programme for available organs. Nevertheless, the number of islet isolations performed in Geneva has increased from an average of 20 a year before GRAGIL to 37 a year since GRAGIL has come into operation.

The cold ischaemia time was on average $6.1 \mathrm{~h}$, and never exceeded $8 \mathrm{~h}$. Although injury could have occurred when islets were transported between isolation and transplantation centres, no clear difference in metabolic outcome was observed between the centres in which transplantations were carried out. In vitro data support the procedure of shipping islet grafts, as good viability and functionality have been described following prolonged islet culture at room temperature [15]. In agreement with this, our data did not indicate that culturing islets for 1 day or for longer periods of time affected the metabolic outcomes although the best results were achieved when islets were cultured for 1 single day in patients \#6 and 9.

The beneficial impact of resuming beta-cell function was shown by a reduction in $\mathrm{HbA}_{1 \mathrm{c}}$ values and insulin requirements among the 5 successful cases. Moreover, two patients achieved insulin independence. 
The explanations for the five islet failures is not clear. The different mechanisms that could be involved have been extensively reviewed elsewhere [16-17]. However, a recently reported series where seven consecutive patients achieved insulin independence and no graft loss after a mean follow-up of 10 months indicates that the islet mass and the immunosuppression strategy probably play a key role in the graft outcome [6]. Patient \#8 received the lowest islet mass (4500 IEQ $/ \mathrm{kg}$ ), one of the two islet preparations scheduled for this patient being discarded on transplantation day because of the retrospective detection of intercurrent bacterial contamination of the donor. Patients $\# 1,3$ and 10 could be considered to have received a borderline islet mass $(7500 \mathrm{IEQ} / \mathrm{kg})$, compared with the rest of the series, while the high islet mass received by patient \#2 was extensively cultured. Of note, patients \#6 and 9 who have had the best metabolic outcome so far also received the highest islet mass of the series, from a single donor, with a culture period under $24 \mathrm{~h}$. All patients had an increase in insulin needs in the period immediately following transplantation. This can be explained by the high steroid dosage used. Similarly, the toxicity of calcineurin inhibitors could have played a role in the progressive beta-cell failure observed in five of our patients. In the absence of any efficient marker, allorejection or autommunity recurrence cannot be ruled out. The role of autoimmune recurrence in the outcome of islet transplantation is disputed [18, 19]. Patient \#10 exhibited positive islet cell antibodies and an islet function failure by the third month. Patient \#8 developed anti-GAD and IA-2 antibodies and lost graft function 3 months after transplantation. On the other hand, despite the presence of positive IA-2 antibodies before transplantation, patient \#9 became insulin-independent.

In summary, we have shown that a multicentre network is an efficient way to improve organ availability for an islet isolation facility. Such an organization achieves the same results as single centres as reported to the International Registry in spite of increased organ and islet transportation time. This network, with its large donor and recipient pool, will allow the investigation of new clinical strategies.

Acknowledgements. We are indebted to all the procurement teams of the University Hospitals in Switzerland and France for their continuous effort and expert assistance in these clinical islet transplantation programme.

The GRAGIL group: B. Boillot, J-L. Descotes, J-J. Rambeaud, D. Masson, D. Halec, C. Sengel, F. Thony, S. Halimi, P. Vialtel; N. Lefrançois, C. Julien, P-J. Valette; M. Pinget, P. Wolf, B. Moulin, J. Tongio, M-H. Tongio, M. Greget, V. Fuss, C. Becker, B. Heyd, J. Chabod, J-M. Rebibou, Y. Hudel, I. Carrey; J. Philippe, C. Becker, R. Mage, F. Triponez, C. Goumaz.

This programme was supported by grants from France's Ministry of Health (PHRC), Alfediam Novo-Nordisk, Region Rhône-Alpes (Emergence), Eli Lilly France.

\section{References}

1. Brendel MD, Hering B, Schultz AO, Bretzel RG (1999) International islet transplant registry. Newsletter 8

2. Alejandro R, Lehmann R, Ricordi C et al. (1997) Longterm function (6 years) of islet allografts in Type I diabetes. Diabetes 46: 1983-1989

3. Secchi A, Socci C, Maffi P et al. (1997). Islet transplantation in IDDM patients. Diabetologia 40: 225-231

4. Keymeulen B, Ling Z, GorusF et al (1998) Implantation of standardized beta-cell grafts in a liver segment of IDDM patients: graft and recipient characteristics in two cases of insulin-independence under maintenance immunosuppression for prior kidney graft. Diabetologia 41 : 452-459

5. Oberholzer J, Triponez F, Mage R et al. (2000) Human islet transplantation: lessons from 13 autologous and 13 allogeneic transplantations. Transplantation 69: 1115-1123

6. ShapiroAM, Lakey JR, Ryan EA et al. (2000) Islet Transplantation in Seven Patients with Type I diabetes mellitus using a glucocorticoid-free immunosuppressive regimen. N Engl J Med 343: 230-238

7. Ricordi C, Gray DW, Hering BJ et al. (1990) Islet isolation assessment in man and large animals. Acta Diabetol Lat 27: 185-95

8. Benhamou PY, Mullen Y (1994) Immunomodulation of Pancreatic Islets by Culture. In: Lanza RP, Chick WL (eds) Pancreatic Islet Transplantation Series Volume 2 CRC Press and Mosby Year Book, Landes RG pp 99-109

9. Bretzel RG, Brandhorst V, Brandhorst H et al. (1999) Improved survival of intraportal pancreatic islet cell allografts in patients with type- 1 diabetes mellitus by refined peritransplant management. J Mol Med 77: 140-143

10. Hanfelt JJ, Slack RS and Gehan EA (1999) A modification of Simon's optimal design for phase II trials: the criterion is median sample size. Control Clin Trials 20: 555-566

11. Benhamou PY, Watt PC, Mullen Y et al. (1994) Human islet isolation in 104 consecutive cases. Factors affecting isolation success. Transplantation 57: 1804-1810

12. Pattou F, Vantyghem MC, Noel C et al. (2000) Sequential intraportal islet allografts in immunosuppressed type I diabetic patients: preliminary results. Transplant Proc 32: 391-392

13. Zeng Y, Torre MA, Karrison T, Thistlethwaite JR (1994) The correlation between donor characteristics and the success of human islet isolation. Transplantation 57: 954-958

14. Brandhorst D, Brandhorst H, Hering BJ, Federlin K, Bretzel RG (1995) Islet isolation from the pancreas of large mammals and humans: 10 years of experience. Exp Clin Endocrinol Diabetes 103 [Suppl 3]: 3-14

15. Nakagawa Y, Kenmochi T, Miyamoto M et al.(1995) Superiority of RPMI 1640 medium for packaging and transportation of islets at ambient temperature. Transplant Proc 27: 3259-3261

16. Hering BJ, Ricordi C (1999) Islet Transplantation for Patients with Type I diabetes. Graft: $12-27$

17. Rosenberg L (1998). Clinical islet cell transplantation. Are we there yet? Int J Pancreatol 24: 145-168

18. Jaeger C, Brendel MD, Hering BJ, Eckhard M, Bretzel RG (1997) Progressive islet graft failure occurs significantly earlier in autoantibody-positive than in autoantibody-negative IDDM recipients of intrahepatic islet allografts. Diabetes 46: 1907-1910

19. Roep BO, Stobbe I, Duinkerken G et al. (1999) Auto- and alloimmune reactivity to human islet allografts transplanted into Type I diabetic patients. Diabetes 48: 484-490 